PACS 68.35Fx, 68.55Ln, 82.65.Dp

\title{
The influence of surface defects on the pinhole formation in silicide thin film
}

\author{
I.V. Belousov ${ }^{1}$, A.N. Grib ${ }^{2}$, G.V. Kuznetsov ${ }^{1}$ \\ ${ }^{1}$ Taras Shevchenko Kyiv National University, 64, Volodymyrska str., 01033 Kyiv, Ukraine \\ ${ }^{2}$ Kharkiv National University, 4, Svobody sq., 61077 Kharkiv, Ukraine
}

\begin{abstract}
The growth of the CoSi layer was considered within the framework of the grain boundary diffusion model. The time dependences of the temperature due to the exothermic reaction of silicide formation as well as the dependences of the CoSi layer thickness were calculated for various values of the reaction activation energy. It was shown that the heat release at high reaction velocities can lead to the considerable increase of the temperature up to melting of the silicide and covering Co layers. The model of pinhole formation in cobalt silicide films was proposed on the basis of local melting in the reaction area at crystal defects of the silicon surface.
\end{abstract}

Keywords: formation of silicide, activation energy, zone of reacting, local melting, defects on surface.

Manuscript received 05.04.06; accepted for publication 23.10.06.

\section{Introduction}

Thin films of $\mathrm{CoSi}_{2}$ have a wide application both in the semiconductor technology and for buffer layers to grow new materials such as high-temperature superconductors due to their good epitaxial and electrical properties [1-8]. However, the principal difficulty in obtaining films with needed properties remains the problem of film roughness (formation of pinholes) [2]. To solve this problem, considerable efforts were applied but the reason of origin of this pinhole formation has not been found till now. It was shown that pinhole formation was promoted by decreasing the surface and interface energies [3]. On the other hand, experimental investigations show that the kinetic processes are important in this case [2]. The supposition was made that some diffusion channels are activated in the film, permitting a significant mass transport to the surface during the rapid thermal process [2]. Moreover, the density of pinholes correlates with the density of structural defects on the Si surface [1]. These experimental data allow to suppose that structural defects play a considerable role in the pinhole formation. Due to the high diffusion coefficient inside the defect and the high reaction ability of atoms on the walls, a considerable increase of the layer growth velocity can be expected. Besides, increasing the temperature owing to exothermic reactions of silicide formation have to be taken into account. Investigations show that $\mathrm{Co}_{2} \mathrm{Si}, \mathrm{CoSi}$ and $\mathrm{CoSi}_{2}$ layers can be formed in silicide [4]. Formation of these chemical compositions is an exothermic reaction with a considerable heat effect [5]. At high diffusion coefficients and velocities of the chemical reaction, this heat release can result in increase of the temperature in the silicide layer.

In this work, we describe the formation of the silicide layer in a two-dimensional structural defect such as a boundary of a grain or a dislocation. We show that Co diffusion into the defect leads to growing the heat release and to melting both the silicide and Co layers due to the low activation energy for the silicide formation reaction. The melted alloy is extracted by capillary forces of spills onto the surface of the Co film and crystallizes creating $\mathrm{CoSi}_{2}$. This process of the pinhole formation can exist when the thickness of the Co layer reaches up to $10 \mathrm{~nm}$.

\section{The model}

The investigation of nucleation stage of the cobalt silicide phases revealed the local formation of the crystallites of the silicide phase surrounded by the unreacted Co film (Fig. 1a, b, d, e). The local origin of the Co silicide phases was found in structural defects on the silicon surface at the initial stage of the $\mathrm{Co}-\mathrm{Si}$ interaction [1]. The coalescence of the lateral cobalt silicide crystallites is supposed to be the preferable mode of the growth during the silicide layer formation on the $\mathrm{Si}$ surface with high density of structural defects (Fig. 1d, e). Therefore, account must be taken of the diffusion processes into the crystal defects at the $\mathrm{Co} / \mathrm{Si}$ interface for the adequate description of the silicide phase growth. The Fisher model [9-11] allows to describe a diffusion process in two-dimensional defects such as the grain boundaries or dislocations. In the 
frames of this model, the defect is considered as the plate with high diffusion coefficient which is implanted between boundaries of the bulk Si material (Fig. 2). The Co atoms penetrate into the defect (plate) from the top thin Co film on the silicon and forming the Co-Si mixture. For simplicity, suppose that the chemical reaction $\mathrm{Co}+\mathrm{Si}=\mathrm{CoSi}$ occurs only at the interface between $\mathrm{Co}-\mathrm{Si}$ mixture and silicon inside the plate; the opposite Si atom flux in the top Co film is neglected. In the present model, the system is instantly heated from the room temperature up to $T_{0}=1000 \mathrm{~K}$ when the $\mathrm{Co}+\mathrm{Si}$ chemical reaction begins.

According to the Fisher model, for the diffusion of Co atoms into the dislocation the displacement of atoms $x$ (Fig. 2) is proportional to the fourth root of time $t$ :

$$
\begin{aligned}
& x=A t^{1 / 4}, \\
& A=\sqrt{\frac{\delta D^{\prime} \sqrt{\pi}}{2 \sqrt{D}}},
\end{aligned}
$$

where $\delta$ is the thickness of the plate, $D$ is the Co diffusion coefficient in the bulk material $(\mathrm{Si}) . D^{\prime}$ is the diffusion coefficient of $\mathrm{Co}$ atoms into the plate as well as into the Co-Si mixture formed into the plate. As the silicide is formed between grains it is assumed that $D^{\prime} / D \sim 10^{4} \ldots 10^{5}[11]$.

The diffusion time of $\mathrm{Co}$ atoms between the $\mathrm{Co} / \mathrm{Co}-\mathrm{Si}$ and $\mathrm{Co}-\mathrm{Si} / \mathrm{Si}$ interfaces in Fisher's model is determined from Eq. (1). The full time of the layer growth is the sum of the time of diffusion and the time of the chemical reaction:

$$
t=\frac{x^{4}}{A^{4}}+\frac{x}{v^{\prime}}
$$

where $v^{\prime}$ is the velocity of the chemical reaction $\mathrm{Co}+\mathrm{Si}$ after Co atom crossed the boundary between Co-Si mixture and $\mathrm{Si}$. The thickness of the layer after the arbitrary time $t$ can be found as the root of the fourth degree from Eq. (2):

$$
x^{4}+\frac{A^{4}}{v^{\prime}} x-A^{4} t=0
$$

The velocity of the layer growth $V$ is also found from equation (2) by differentiation:

$$
V=\frac{d x}{d t}=\left(\frac{4 x^{3}}{A^{4}}+\frac{1}{v^{\prime}}\right)^{-1}
$$

The limiting cases of (4) are $V=v^{\prime}$ if $4 x^{3} v^{\prime}<<A^{4}$ and $V=\frac{a^{4}}{4 x^{3}}$ at $4 x^{3} v^{\prime}>>A^{4}$. The change of these cases occurs when $x^{\prime} \approx \sqrt[3]{\frac{A^{4}}{4 v^{\prime}}}$. Comparing this expression with the characteristic thickness $x_{1}$ of changing the growth mechanisms, which follows from the usual diffusion [12-15] $x_{1} \approx \frac{D}{v}$ ( $v$ is the constant of layer growth in the kinetic regime for the homogeneous layer), we can see that at large $v$ and $v^{\prime}$ the first expression allows to get much more thickness than in the usual case. For example, at $D \approx 10^{-14} \mathrm{~m}^{2} / \mathrm{s}, \delta \approx 10^{-8} \mathrm{~m}$ and $D^{\prime} \approx$ $10^{-10} \mathrm{~m}^{2} / \mathrm{s}$ we obtain $A \approx 3 \cdot 10^{-6}$ that at $v^{\prime} \approx 10^{-2} \mathrm{~m} / \mathrm{s}$ gives $x^{\prime} \approx 1.3 \cdot 10^{-7} \mathrm{~m}$ instead of $x_{1} \approx 1 \cdot 10^{-12} \mathrm{~m}$ at the same value of $v$. The high velocities of the chemical reaction can exist due to the dependence of the activation energy on the density of the surface charge [12] which can be high within the boundaries of the structural defects or Si cracks.

Let us estimate the temperature of the CoSi layer during the exothermic reaction $\mathrm{Co}+\mathrm{Si}=\mathrm{CoSi}$ at the $\mathrm{CoSi} / \mathrm{Si}$ interface. Consider the case when this interface has the width $\delta$ and is placed between the boundaries of the defect. Assuming a low thickness of Co layer above the defect $(\sim 1 \mathrm{~nm})$ and supposing that $t \leq 10^{-7} \mathrm{~s}$ (the distance of temperature diffusion $x^{\prime \prime}=2 \sqrt{a t}$ is lesser than the thickness of the Si sample ( $a$ is the temperature conductivity coefficient, $a \approx 10^{-5} \mathrm{~m}^{2} / \mathrm{s}$ ) and we can consider the quasi-one-dimensional case of heating the edge of the Si half-infinite rod. The functional solution of the thermal conductivity equation in this case gives the temperature on distance $r$ from the $\mathrm{CoSi} / \mathrm{Si}$ interface at chosen moment $\tau$ [16]:

$T(r, \tau)=T_{0}+\frac{2}{K_{\varepsilon}} \int_{0}^{\tau} \frac{q[T(0, z)] e^{-\frac{r^{2}}{4 a(\tau-z)}}}{\sqrt{\pi(\tau-z)}} d z$,

where $K_{\varepsilon}=\sqrt{\lambda_{2} c_{2} \rho_{2}}$, with the thermal conductivity $\lambda_{2}$, the heat capacity $c_{2}$ and density $\rho_{2}$ of $\mathrm{Si} ; q[T(0, z)]$ is the heat flux in $\mathrm{J} / \mathrm{m}^{2}$ on the $\mathrm{CoSi} / \mathrm{Si}$ interface due to the exothermic reaction:

$q[T(0, \tau)]=\widetilde{Q} \rho_{1} V(T, \tau)$,

$\rho_{1}$ is the concentration of Co at the interface, $\widetilde{Q}$ is the heat effect of the reaction. The temperature at the interface can be obtained from this relation:

$T(0, \tau)=T_{0}+\frac{2}{K_{\varepsilon}} \int_{0}^{\tau} \frac{q[T(0, z)]}{\sqrt{\pi(\tau-z)}} d z$.

The change of the factor $\frac{r^{2}}{4 a \tau}$ is small, and further we will suppose that the interface does not move in the process of the reaction. Then, we can solve the system (3), (4), (6), (7) using the numerical method proposed in $[17,18]$ for $\tau_{m-1}<z<\tau_{m}$ : 

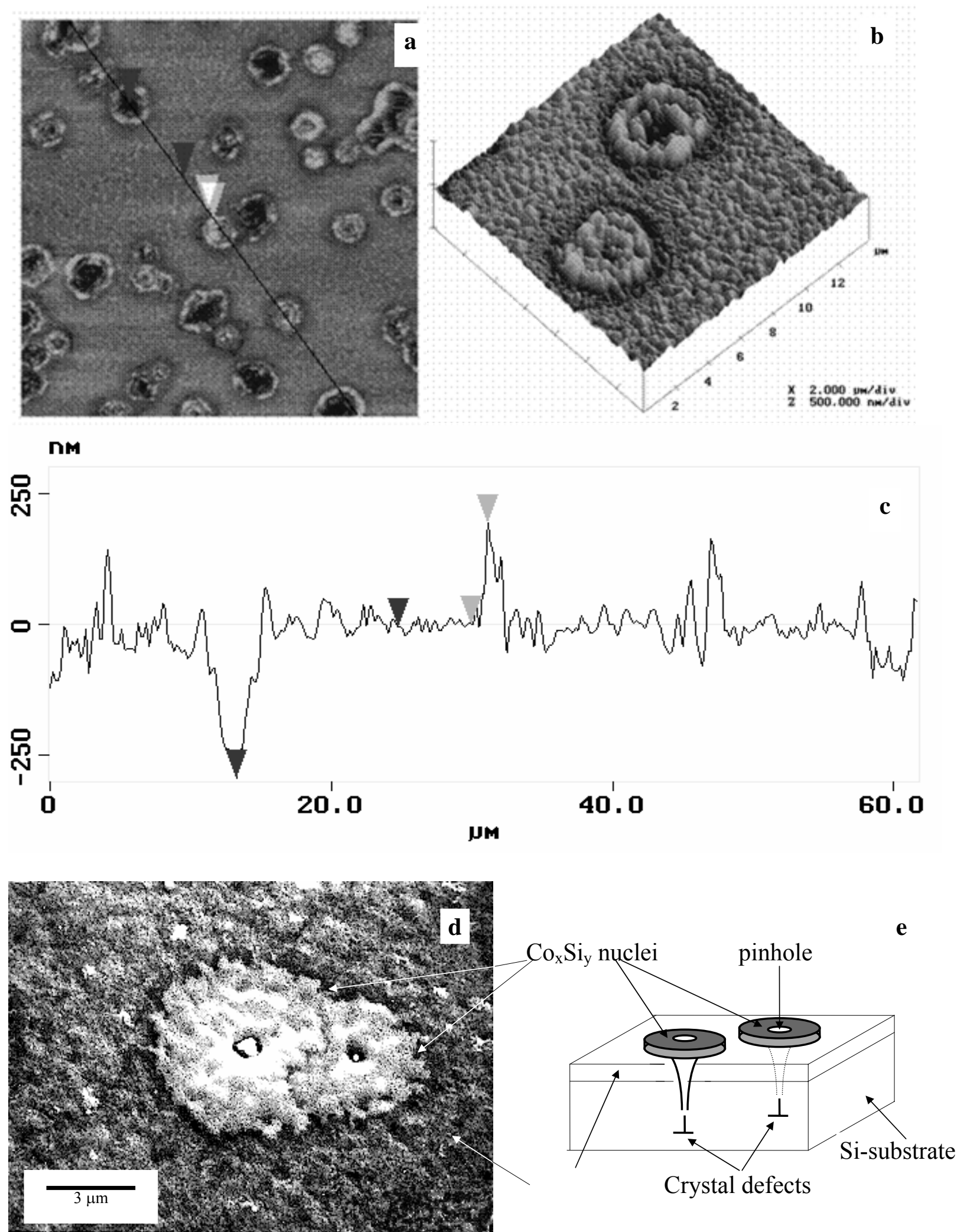

Fig. 1. AFM images (a, b), surface profile (c) and SEM image (d) for the $\mathrm{Co} / \mathrm{Si}$ structure after vacuum annealing at $873 \mathrm{~K}$. The scheme of the lateral silicide crystallites and pinholes formation (e). 
$T\left(0, \tau_{m}\right)=T_{0}+\frac{2}{K_{\varepsilon}} \int_{0}^{\tau_{m-1}} \frac{q[T(0, z)]}{\sqrt{\pi(\tau-z)}} d z+$

$+\frac{2 q\left[T\left(0, \tau_{m-1}\right)\right]}{K_{\varepsilon}} \int_{\tau_{m-1}}^{\tau_{m}} \frac{1}{\sqrt{\pi(\tau-z)}} d z$.

The temperature dependence of the velocity of layer growth is determined by the temperature dependences of the velocity constants

$v=v_{0} e^{-\frac{U_{r}}{k T}}, v^{\prime}=v_{0}^{\prime} e^{-g \frac{U_{r}}{k T}}$

and coefficients of diffusion:

$D=D_{0} e^{-\frac{U_{d}}{k T}}, D^{\prime}=D_{0}^{\prime} e^{-\frac{U_{\mathrm{def}}}{k T}}$,

where $v_{0}, v_{0}^{\prime}, D_{0}, D_{0}^{\prime}$ are pre-exponential factors, $U_{r}$ is the activation energy of the reaction, $U_{d}$ is the diffusion energy barrier, $U_{\text {def }}$ is the energy barrier on the diffusion inside the defect, $g$ is a factor $0<g<1$.

\section{Results and discussion}

The dependences $D(T)$ and $D^{\prime}(T)$ were chosen as follows: $D=10^{-5} e^{-\frac{1.9 \mathrm{eV}}{k T}}, \quad D^{\prime}=10^{-5} e^{-\frac{3}{5} \cdot \frac{1.9 \mathrm{eV}}{k T}}$. The value $U_{d}=1.9 \mathrm{eV}$ was experimentally found in [4] for the CoSi layer growth. Because there are no experimental data on the linear growth of layer in $\mathrm{Co}-\mathrm{Si}$ system, we used the expression $v^{\prime}=10^{3} e^{-g \frac{1.9 \mathrm{eV}}{k T}}$, which at $1000 \mathrm{~K}$ and $g=1$ gives the same velocity $10^{-7} \mathrm{~m} / \mathrm{s}$ as was found experimentally for the system Ni-Si [19]. Using the factor $g$ in this expression, we can model the change of the reaction activation energy due to the surface charge inside the defect. The initial temperature $T_{0}=1000 \mathrm{~K}$ was chosen. The energy effect of the

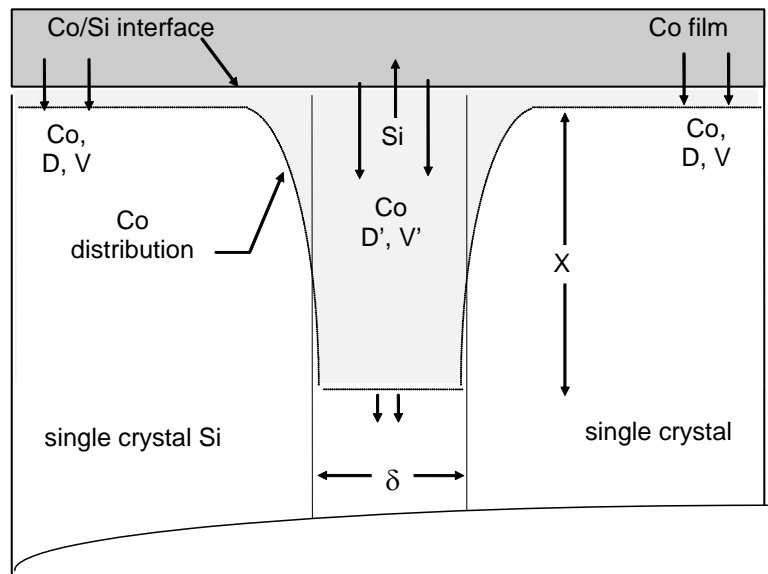

Fig. 2. Fisher's model for the diffusion of Co atoms into the dislocation and Co atom displacement $x$. reaction $\widetilde{Q}$ is equal to $1.15 \cdot 10^{6} \mathrm{~J} / \mathrm{kg}$ [5]. Dependences $T(0, t)$ are shown in Fig. 3 for various values of $g$ and $\delta$ parameters. At small $\delta$, the sufficient exponential growth of the interface temperature is reached only at great velocities (or small values of $g$, Fig. 3a, curves 1,2). Then the temperature decreases due to the transfer to the diffusion growth of the layer. The decrease of the temperature obeys the $t^{-1 / 4}$ law accordingly to the chosen model. At larger values of $g$, the growth of the layer is slow (Fig. 3a, curves 3,4), and the temperature decreases after peaking which does not reach the melting point of Si $(\sim 1696 \mathrm{~K})$.
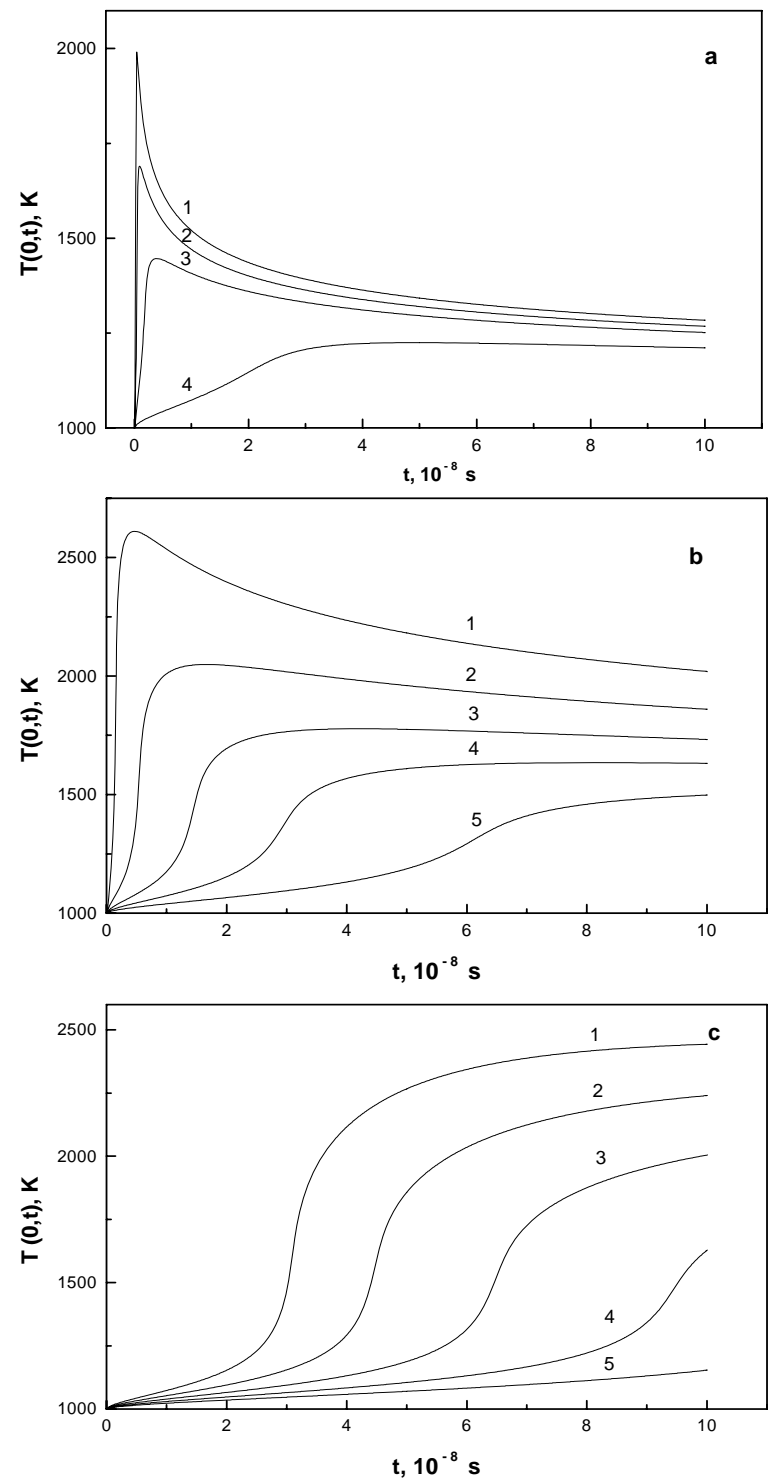

Fig. 3. Dependences of the $\mathrm{CoSi} / \mathrm{Si}$ interface temperature $T(0, t)$ on time at various values of the parameters $\delta$ and $g$ : a) $\delta=10^{-8} \mathrm{~m}, g=0.18(1), 0.25(2), 0.32(3), 0.40(4)$; b) $\delta=$ $=10^{-7} \mathrm{~m}, g=0.30(1), 0.35(2), 0.38(3), 0.40(4), 0.42(5)$; c) $\delta=5 \cdot 10^{-7} \mathrm{~m}, \quad g=0.40(1), 0.41(2), 0.42(3), 0.43(4)$, 0.44 (5). Lines denote the $\mathrm{CoSi}$ melting temperature $T_{\text {melt }}=1737 \mathrm{~K}$ 

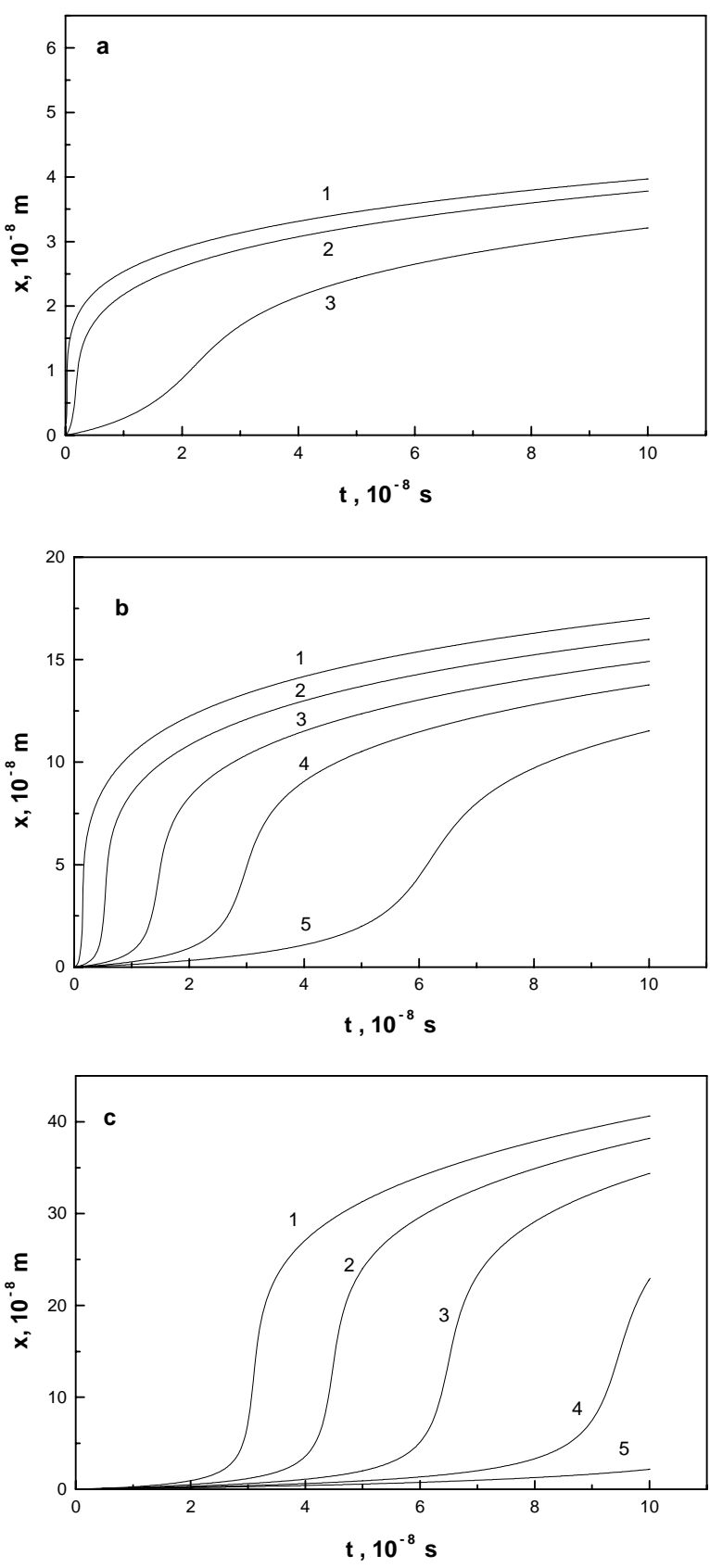

Fig. 4. Dependences of the CoSi layer thickness $x$ on time at various values of the parameters $\delta$ and $g$ : a) $\delta=10^{-8} \mathrm{~m}, g=$ 0.18 (1), 0.32 (2), 0.40 (3); b) $\delta=10^{-7} \mathrm{~m}, g=0.30(1)$, $0.35(2), 0.38(3), 0.40(4), 0.42(5) ; \mathrm{c}) \delta=5 \cdot 10^{-7} \mathrm{~m}, g=$ $=0.40(1), 0.41(2), 0.42(3), 0.43(4), 0.44(5)$.

Increasing the parameter $\delta$ leads to increase of $g$ at which the transition to high temperatures occurs (Figs $3 b, c)$. The peak in the $T(t)$ dependences is shifted to large times and decrease of the temperature is moderate. The range of the parameters $g$ in which the change of temperature regimes appears is reduced $\left(\Delta g \approx 0.2\right.$ at $\delta=10^{-8}, \Delta g \approx 0.12$ at $\delta=10^{-7}$ and $\Delta g \approx 0.04$ at $\delta=5 \cdot 10^{-7}$ ). The dependence of the thickness of the
CoSi layer $x$ on time is shown in Fig. 4. At $\delta=10^{-8}$ and small $g$, the thickness obeys the $t^{1 / 4}$ law, as it follows from Eq. (3) (Fig. 4a, curve 1). While the parameter $g$ increases, the dependence $x(t)$ turns to the exponential growth at small $t$ and $t^{1 / 4}$ growth at large $t$ (Fig. 4a, curves 2,3$)$. The increase of the parameter $\delta$ leads to significant enhancement of the layer thickness (Fig. 4b, c).

The temperature of the interface can reach the melting temperatures of $\mathrm{CoSi}, \mathrm{Si}$ and Co especially at larger values of $\delta=(1 \ldots 5) \cdot 10^{-7} \mathrm{~m}$ (Fig. 3b, c). If the activation energy of the reaction is small, the temperature in the reaction area at the interface increases to very high values (Fig. 3b, curves 1-3) and decays with small decrement, which leads to melting of the $\mathrm{CoSi}$ layer during this time. Moreover, the Co layer with the thickness $\sim 10 \mathrm{~nm}$ will be melted too, accordingly to the chosen model of heating of the half-infinite rod. At larger thickness of the Co layer, the exponent in the equation (5) has to be taken into account, and the temperature of the Co layer will be lower than that inside the defect. Note that, when the temperature of the reaction area will reach the melting points of $\mathrm{Co}, \mathrm{Si}$ and CoSi phases, the chosen model will not describe heating the interface because the specific heats of melting do not take into consideration. It means that the temperature in the reaction area will not increase higher then in melting points or will decrease when the reaction will be finished. Calculations show that the melted $\mathrm{CoSi}$ is extracted by capillary forces on the Co surface for $\sim 10^{-7} \mathrm{~s}$ and after the process of crystallization $\mathrm{CoSi}_{2}$ is formed on the top Co film. Thus, the melting of the CoSi layer arises during the exothermic reaction because of the small activation energy of the reaction in the structural defect and as a result of this local melting process the pinhole channel is formed.

\section{Conclusions}

We considered the growth of the cobalt silicide layer in the frames of the model of grain boundary diffusion. The diffusion front of $\mathrm{Co}$ in this model shifts obeying the $t^{1 / 4}$ law. Because of this law, the layer thickness can grow up to $0.1 \mu \mathrm{m}$ at large velocities of the chemical reaction. This growth rate leads to a great extraction of heat due to the exothermic reaction at the $\mathrm{CoSi} / \mathrm{Si}$ interface. We calculated the time dependence of the temperature of the reaction area at the interface $\mathrm{CoSi} / \mathrm{Si}$ and found that for $t \sim\left(10^{-8} \ldots 10^{-7}\right) \mathrm{s}$ the temperature can increase to the melting points of $\mathrm{Co}, \mathrm{Si}$ and $\mathrm{CoSi}$. The liquid alloy spills out on the top of the Co film and pinholes are formed into silicide film as well as lateral silicide crystallites.

\section{References}

1. I. Belousov, E. Rudenko, S. Linzen, P. Seidel // Thin Solid Films, 325, p. 145 (1998). 
2. Like Ruan and D.M. Chen // Appl. Phys. Lett. 72, p. 3464 (1998).

3. R.T. Tung // Mater. Chem. Phys. 32, p. 107 (1992).

4. S.S. Lau, J.W. Mayer, K.N. Tu // J. Appl. Phys. 49, p. 4005 (1978).

5. P.V. Geld, F.A. Sidorenko, Silicides of transition metals of fourth group. Metallurgia, Moscow, 1971 (in Russian).

6. M.L. Lee and P.A. Bennett // Phys. Rev. Lett. 75, p. 4460 (1995).

7. G.L. Van Gurp, C. Langereis // J. Appl. Phys. 46, p. 4301 (1975).

8. G. Ottaviani, M. Costato // J. Crystal Growth 45, p. 365 (1978).

9. J.C. Fisher // J. Appl. Phys. 22, p. 74 (1951).

10. J.P. Stark // J. Appl. Phys. 36, p. 3938 (1965).

11. Paul G. Shewmon, Diffusion in solids. McGraw Hill Book Co., Inc., NY, 1992.
12. V.N. Chebotin, Physical chemistry of solids. Khimiyay, Moscow, 1982 (in Russian).

13. V.I. Dybkov, Kinetics of solid phase chemical reaction. Naukova Dumka, Kiev,1992 (in Russian).

14. U. Gossele, K.N. Tu // J. Appl. Phys. 53, p. 3252 (1982).

15. Ya.E. Geguzin, The diffusion zone. Nauka, Moscow, 1979 (in Russian).

16. A.V. Lykov (ed.), Methods of registration of thermal conductivity and temperature conductivity. Energy, Moscow, 1973 (in Russian).

17. A.V. Grosbie, R. Viskanta // Intern. J. Heat and Mass Transfer 11, p. 2 (1969).

18. N.M. Belyaev, A.A. Ryadno, Methods of theory of thermal conductivity. Visshaya Shkola, Moscow, 1982 (in Russian).

19. D.J. Coe and E.H. Roderick // J. Phys. D 9, p. 965 (1976). 